\title{
Two-Dimensional Nanoplatelet Superlattices Overcoming Light Outcoupling Efficiency Limit in Perovskite Quantum Dot Light-Emitting Diodes
}

\section{Sudhir Kumar}

ETH Zürich

\section{Tommaso Marcato}

ETH Zürich

Frank Krumeich

ETH https://orcid.org/0000-0001-5625-1536

Yen-Ting Li

NTUST Taipei, Taiwan and NSRRC Hsinchu, Taiwan

Yu-Cheng Chiu

National Taiwan University of Science and Technology, Taipei, Taiwan

Chih-Jen Shih ( $\square$ chih-jen.shih@chem.ethz.ch )

ETH Zürich https://orcid.org/0000-0002-5258-3485

\section{Article}

Keywords: Quantum dot light-emitting diodes, LEDS, TDM

Posted Date: May 17th, 2021

DOI: https://doi.org/10.21203/rs.3.rs-344894/v1

License: () (1) This work is licensed under a Creative Commons Attribution 4.0 International License. Read Full License

Version of Record: A version of this preprint was published at Nature Communications on April 19th, 2022. See the published version at https://doi.org/10.1038/s41467-022-29812-5. 


\title{
Two-Dimensional Nanoplatelet Superlattices Overcoming Intrinsic Light Outcoupling Efficiency Limit in Perovskite Quantum Dot Light-Emitting Diodes
}

\author{
Sudhir Kumar ${ }^{1 \dagger}$, Tommaso Marcato ${ }^{1 \dagger}$, Frank Krumeich ${ }^{2}$, Yen-Ting $\mathrm{Li}^{3,4}$, Yu-Cheng $\mathrm{Chiu}^{3}$, and \\ Chih-Jen Shih ${ }^{1 *}$
}

\author{
${ }^{1}$ Institute for Chemical and Bioengineering, ETH Zürich, 8093 Zürich, Switzerland \\ ${ }^{2}$ Laboratory of Inorganic Chemistry, ETH Zürich, 8093 Zürich, Switzerland \\ ${ }^{3}$ Department of Chemical Engineering, National Taiwan University of Science and Technology, Taipei 10607, Taiwan. \\ ${ }^{4}$ National Synchrotron Radiation Research Center, Hsinchu 30076, Taiwan. \\ tThese authors contributed equally. \\ *All correspondence should be addressed to chih-jen.shih@chem.ethz.ch
}

\begin{abstract}
Quantum dot (QD) light-emitting diodes (LEDs) are emerging as one of the most promising candidates for next-generation displays. However, their intrinsic light outcoupling efficiency remains considerably lower than the organic counterpart, because it is not yet possible to control the transition-dipole-moment (TDM) orientation in QD solids at device level. Here, using the colloidal lead halide perovskite nanoplatelets (NPLs) as a model system, we report a directed self-assembly approach to form the two-dimensional superlattices (2DSLs) with the out-of-plane vector perpendicular to the substrate plane. The ligand and substrate engineering yields close-packed planar arrays with the side faces linked to each other. Emission polarization in individual NPLs rescales the radiation from horizontal and vertical transition dipoles, effectively resulting in preferentially horizontal TDM orientation. Based on the emissive thin films comprised of stacks of 2D superlattices, we demonstrate an enhanced ratio of horizontal dipole as revealed by 2D $k$-space spectroscopy. Our optimized single-junction QD LEDs showed peak external quantum efficiency of up to $24.2 \%$ and power efficiency exceeding $110 \mathrm{Im} \mathrm{W}^{-1}$, comparable to state-of-the-art organic LEDs.
\end{abstract}




\section{Introduction}

Quantum dot (QD) light-emitting diodes (LEDs) are ideal for next-generation flat-panel displays because of their scalability, cost effectiveness, emission color purity, and tunable chromaticity. ${ }^{1-5}$ Considerable research efforts in the past two decades have demonstrated high-efficiency QD LEDs using CdSe, InP, and lead halide perovskite (LHP) nanocrystals (NCs), with external quantum efficiencies, $\eta_{\text {ext }}$, of up to $20.5 \%,{ }^{4} 21.4 \%,{ }^{6}$ and $23.4 \%,{ }^{7-9}$ respectively. To date, the methods used to enhance device performance have mainly focused on the passivation of NC surface defects. Taking the defect-tolerant LHP QD system ${ }^{10-13}$ as an example, ligand engineering, modulation of stoichiometric compositions, and cation/anion mixing $^{14-18}$ have been employed to substantially improve the device performance. ${ }^{7-9}$ Nevertheless, as the internal quantum efficiency has approached unity, new strategies that boost the intrinsic light outcoupling efficiency, $\eta_{\text {out, }}$, become increasingly attractive to bring the device performance to the next level.

In thin-film LEDs, it is well-known that majority of radiation generated within the emissive layer is trapped inside the thin-film stack through the dissipating pathways of waveguide, surface plasmon, and substrate modes, eventually converting to heat. The classical ray optics theory gives a first approximation, $\eta_{\text {out }}=1 /\left(2 n^{2}\right)$, where $n$ is the refractive index of emissive layer. ${ }^{19}$ For example, in the LHP systems, given $n \sim 2.0-2.6$, one could estimate $\eta_{\text {out }}$ of only $\sim 15 \%$. $^{20-}$ ${ }^{22}$ An approach to address this challenge is to intrinsically increase $\eta_{\text {out }}$ by inducing emission directionality in quantum emitters. For example, in organic LEDs (OLEDs), orienting emissive molecules with their transition dipole moments (TDMs) parallel to the substrate would outcouple more light within the critical angle for the glass-air interface. ${ }^{23-25}$ The major advantage of this approach is to allow large-scale manufacturing without the need of other physical light-extraction techniques, such as microlens arrays, photonic crystals, surface corrugation, and matching index grating, ${ }^{26-28}$ which often demand expensive and complicated fabrication $^{28,29}$ and even compromise emission chromaticity from different viewing angles. ${ }^{28,30}$

Spontaneous emission in organic molecules involves highly localized excitons, which offer clues to the correlation between TDM and molecular orientations that could guide rational molecular design and thin-film processing. ${ }^{25}$ In contrast, excitons in inorganic semiconductor crystals reside in extended band edge states of complex three-dimensional (3D) symmetry, which usually leads to isotropic TDM orientation. An ideal candidate to control the TDM orientation for LEDs is the atomically thin two-dimensional (2D) materials, such as $\mathrm{MoS}_{2}$ monolayer, ${ }^{31}$ because the $2 \mathrm{D}$ electronic structure confines all bright excitons in-plane. ${ }^{32}$ The major challenge is that $2 \mathrm{D}$ monolayers are very sensitive to surface defects that drastically 
quench photoluminescence (PL) by orders of magnitude, particularly at high exciton concentrations, hindering practical applications. ${ }^{33}$

Recent experimental studies have also suggested that excitons in the few-monolayer-thick colloidal CdSe nanoplatelets (NPLs) have completely in-plane TDM orientation. ${ }^{34,35,34,36}$ Indeed, emission in the quantum-confined zinc-blende (ZB) crystal structure comes exclusively from the heavy-hole states, which accidentally have only mixed $p_{x}$ and $p_{y}$ symmetry, forming a bright plane that coincides with the platelet plane. ${ }^{34}$ The CdSe NPLs, however, suffer from relatively low PL quantum yield, $\eta_{\mathrm{PL}}$, and more critically, lose the preferential TDM orientation in their stacks. ${ }^{36}$ To our knowledge, preferentially horizontal TDM orientation in QD-assembled thin films has never been demonstrated at device level.

With the above background in mind, to intrinsically enhance light outcoupling in QD-assembled thin films for high-efficiency LEDs, a challenging list of requirements must be met, including TDM orientation control in individual NCs, controlled NC assembly without compromising $\eta_{\mathrm{PL}}$ and TDM orientation, and ligand engineering that balance dielectric confinement and carrier injection. In this report we show that all these requirements can be satisfied by a scalable $2 \mathrm{D}$ superlattice system comprised of LHP NPLs.

\section{Results and discussions}

Emission polarization in individual NPLs. First, we would like to point out that NPLs do not necessarily possess 2D electronic structure. Unlike the ZB crystal system, the LHP is of cubic structure with octahedral symmetry, where the bandgap occurs at the $\mathbf{R}$ point in the Brillouin zone, isomorphic to the $\Gamma$ point. The spin-orbit interaction leads to the splitting of the conduction band states into a fourfold $\Gamma_{8}^{-}$and a twofold $\Gamma_{6}{ }^{-}$states, and the valence band $\Gamma_{6}{ }^{+}$states are $s^{-}$ like having zero orbital angular momentum. ${ }^{37,38} \mathrm{~A}$ proper description for spontaneous emission in LHP NPLs corresponds to the transition between the $\Gamma_{6}^{-}$and $\Gamma_{6}{ }^{+}$states, which is of mixed $p_{x}, p_{y}$, and $p_{z}$ symmetry (Supplementary Section 1.1). We therefore deduce that, unless LHP NPLs are of atomic thickness that largely changes the band structure, the TDM orientation remains isotropic, in spite of a degree of quantum confinement.

It should be noted that controlling TDM orientation is not the only approach to induce emission directionality in individual QDs. Early findings from single-molecule spectroscopy have explored the effect of emission polarization in anisotropically dielectric-confined nanostructures. ${ }^{39,40}$ Specifically, consider an isolated NC surrounded by low-dielectric-constant ligand medium, with the dielectric constants of $\epsilon_{\mathrm{NC}}$ and $\epsilon_{\mathrm{m}}$, respectively. The local electric field within NC, $\mathbf{E}^{\text {loc }}$, induced by an external field, E, would strongly depend on the NC shape and the dielectric contrast, $\widetilde{\epsilon}=\epsilon_{\mathrm{NC}} / \epsilon_{\mathrm{m}}$, characterized by the local field factors, $f_{\mathrm{i}}=E_{i}^{\mathrm{loc}} / E_{i}$, where 
subscript $i$ correspond to $x, y$, and $z$ coordinates in space (Supplementary Section 1.2). Figure 1a presents a set of calculated ratios of local field factor, $f_{x} / f_{z}$, as a function of the NC aspect ratio, $\mathrm{AR}$, for spheroids and square cuboids, in which $f_{x}=f_{y}$, using the dielectric constants in our synthesized LHP NCs $\left(\epsilon_{\mathrm{NC}}=4.7\right.$ and $\left.\epsilon_{\mathrm{m}}=2.129\right)$. The cubic and spherical NCs $(\mathrm{AR}=1)$ are characterized by their isotropic polarization response, which then departs from unity as the shape evolves towards asymptotic rod $(A R \rightarrow 0)$ and disk $(A R \rightarrow \infty)$. Specifically, the NPL has its in-plane (IP, $x$ or $y$ direction) local field factor increasing with AR, but the other way around for the out-of-plane (OP, $z$ direction) component. When $\mathrm{AR} \rightarrow \infty, f_{x}=1$ and $f_{z}=1 / \widetilde{\epsilon}$ (see Supplementary Section 1.2), giving the theoretical upper limit for $f_{x} / f_{z}=\widetilde{\epsilon}$.

The emission radiative rate for a given dipole is proportional to $(\mathbf{p} \cdot \mathbf{f})^{2}$, where $\mathbf{p}$ and $\mathbf{f}$ are the transition dipole moment and local field factor vectors, respectively (Supplementary section 1.3). As a result, in a square NPL with $f_{x}=f_{y}$, the emission from an OP dipole is significantly screened and that from an IP dipole is amplified, resulting in an enhanced emission directionality towards the OP direction. Under the assumption of isotropic TDM orientation, the effective IP dipole ratio within individual NC determined by far-field measurement, $\Theta_{I P}$, is given by

$$
\Theta_{\mathrm{IP}}=\frac{f_{x}+f_{y}}{f_{x}+f_{y}+f_{z}}
$$

Accordingly, Eq. (1) and Fig. 1a reveal that a cubic NC with $f_{x} / f_{z}=1$ would give $\Theta_{\mathrm{IP}}$ of 0.67 , corresponding to isotropic radiation, but the square LHP NPLs could yield $\Theta_{\text {IP }}$ up to 0.82 for $\mathrm{AR} \rightarrow \infty$ (Supplementary section 1.2), even with isotropic TDM orientation. We notice that the predicted $\Theta_{\mathrm{IP}}$ upper limit nicely agrees with the experimentally measured values in 3monolayer LHP NPLs (thickness $d=1.8 \mathrm{~nm}$ and AR $\sim 30$ ) in which $\Theta_{\mathrm{IP}}=0.81-0.85 .{ }^{41,42}$

The NC-shape-induced emission directionality is further illustrated in Figs. $1 \mathrm{~b}$ and $1 \mathrm{c}$, which compare the calculated electric fields and radiation patterns from horizontal and vertical dipoles. In these simulations, a Hertzian dipole is placed in the center of cubic NC or NPL (AR $=10$ ) embedded in a dielectric film sitting on top of a semi-infinite glass substrate, with the NC $z$ axis perpendicular to the substrate ( $x-y$ plane). The dielectric anisotropy of NPL rescales the oscillator strength for horizontal and vertical dipoles, which yield enhanced and reduced radiation power, respectively. As a result, more light can be directed within the critical angle ( $\sim 1$ degrees; the red angular regions in Fig. 1c) for the glass-air interface. In principle, this scenario would apply to any NC geometry, but overall, square NPLs would be the most effective for LEDs, because of the geometric similarity with the substrate. 


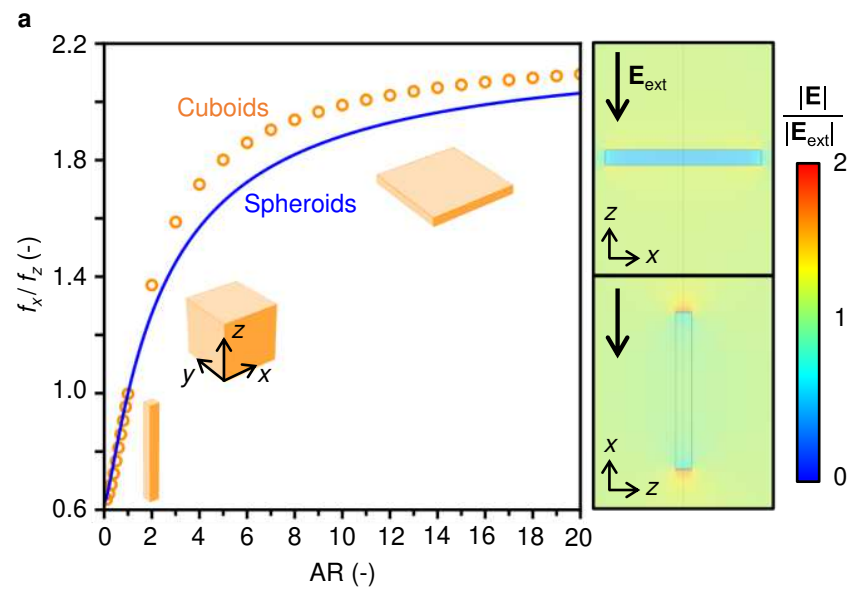

b
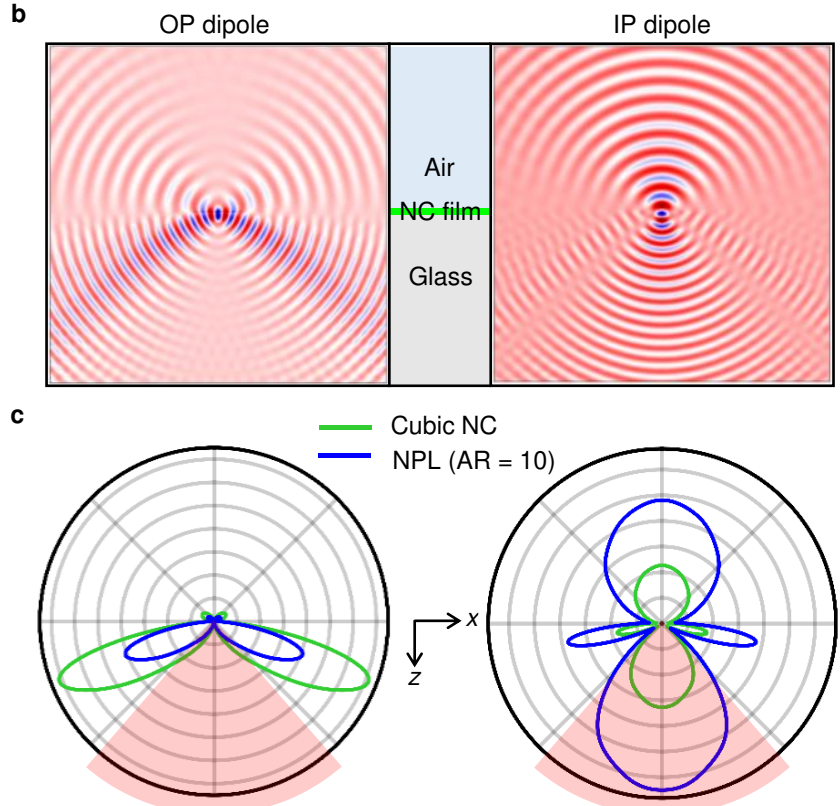

Fig. 1 | Emission polarization in anisotropically dielectric-confined NCs. a, Calculated ratio of horizontal $(x)$ to vertical $(z)$ local field factor, $f_{x} / f_{z}$, as a function of $A R$ considering individual cuboidal and spheroidal perovskite NCs $\left(\epsilon_{\mathrm{NC}}=4.7\right)$ embedded in a low-dielectricconstant ligand $\left(\epsilon_{\mathrm{m}}=2.129\right)$ medium. Right panels present simulated electric field distributions near a representative square NPL of $A R=10$ by applying a constant vertical (top) and horizontal (bottom) external electric field, $\mathbf{E}_{\text {ext }}$, revealing the polarization anisotropy. b-c, Calculated electric field intensity profiles (b) and radiation patterns (c) in the $x-z$ plane for an out-of-plane (OP; left) and in-plane (IP; right) dipole placed in the center of a cubic NC and NPL of $A R=10$ embedded in a dielectric emissive film sitting on the glass substrate. The dielectric anisotropy of NPL rescales the radiation from horizontal and vertical transition dipoles, thereby directing more light within the critical angle (red angular regions) for the glassair interface $(x-y$ plane).

Directed Assembly of NPLs. The physical picture of single-dipole radiation presented in Fig. $1 \mathrm{c}$ is valid at device level only if individual NCs retain their radiation pattern in the assembled thin films. In practice, many undesirable effects, such as quantum resonance ${ }^{43}$ and energy transfer $^{5}$ could come into play upon NC assembly. For example, strong face-to-face interactions between CdSe NPLs in the drop-casted film yield the "edge-on" NPL orientation 
with respect to the substrate, resulting in isotropic radiation pattern. ${ }^{36}$ The emergence of LHP NCs opens an avenue to address the longstanding challenge. Indeed, the LHP dielectric response is high at low frequencies $(\epsilon>20)$, due to the lattice softness that generates strong phonon and molecular contributions, but drops rapidly in the visible regime $(\epsilon \sim 5) .{ }^{44} \mathrm{As}$ a result, the electrostatic interactions between neighboring NCs are significantly screened, largely alleviating the undesirable effects. This is reflected by our recent observations that an ultrathin organic spacer of $\sim 0.67 \mathrm{~nm}$ is sufficient to decouple neighboring NPLs in their superlattices. ${ }^{42}$

We synthesized mixed-cation perovskite NPLs with formula of $\mathrm{FA}_{0.5} \mathrm{MA}_{0.5} \mathrm{PbBr}_{3}$, where $\mathrm{FA}=$ formamidinium, $\mathrm{CH}_{3}\left(\mathrm{NH}_{2}\right)_{2}{ }^{+}$and $\mathrm{MA}=$ methylammonium, $\mathrm{CH}_{3} \mathrm{NH}_{3}{ }^{+}$, using the modified ligandassisted re-precipitation (LARP) method at room temperature (details see Methods). ${ }^{45,46}$ The NPL aspect ratio was tuned and optimized by the ligand concentration and hydrophobicity. ${ }^{35}$ Specifically, the NC ARs can be increased by reducing the ligand hydrophobicity that stabilizes the small crystals, and increasing ligand concentration could access to few-monolayer-thin NPLs with high degrees of quantum confinement. In addition, device considerations, including balanced carrier conductivity and proper NPL dielectric confinement, were also taken into account. ${ }^{46}$ For example, excessively thin NPLs would compromise electrical stability and thinfilm impedance, both lowering the device efficiency. After extensive experimentation, mixed $n$ decylamine (DA) and oleic acid (OA) was chosen as the major molecular ligand unless mention otherwise. We notice that the decyl and oleic tails have similar degree of hydrophobicity. Figure 2a presents the transmission electron microscope (TEM) image for the synthesized square NPLs, having the average lateral length and thickness of $11 \pm 2$ and $4 \pm 1 \mathrm{~nm}$, respectively. Given the TEM-observed AR value ( 2.75), we predict its $\Theta_{\text {IP }}$ value to be 0.74 .

Figures $2 \mathrm{e}-2 \mathrm{j}$ compare the synchrotron grazing-incidence wide-angle and small-angle $\mathrm{X}$-ray scattering (GIWAXS and GISAXS) patterns for the representative emissive thin films (thickness $t \sim 30 \mathrm{~nm}$ ) studied here. Direct drop casting of colloidal solution on glass yields NPL solid with nearly random orientation (Fig. 2e), exhibiting the Debye-Scherrer (DS) rings corresponding to the intracrystal perovskite structure, denoted (100), (110), and (200). Shearinduced ordering during spin coating leads to the formation of superstructure with an improved degree of alignment for the NPL OP vector (Fig. 2f). The emergence of (100) Bragg peak along the $q_{z}$ axis and the vanishing (110) DS ring suggest that the majority of NPLs was horizontally oriented with the OP vector perpendicular to the substrate plane. 


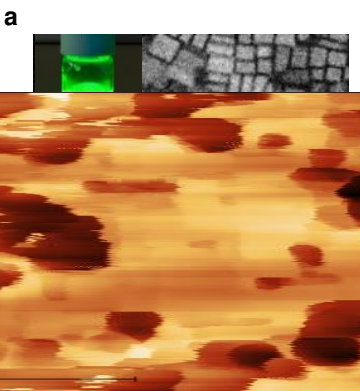

e

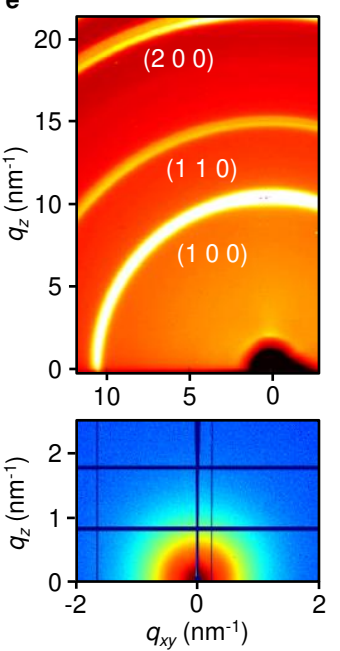

b
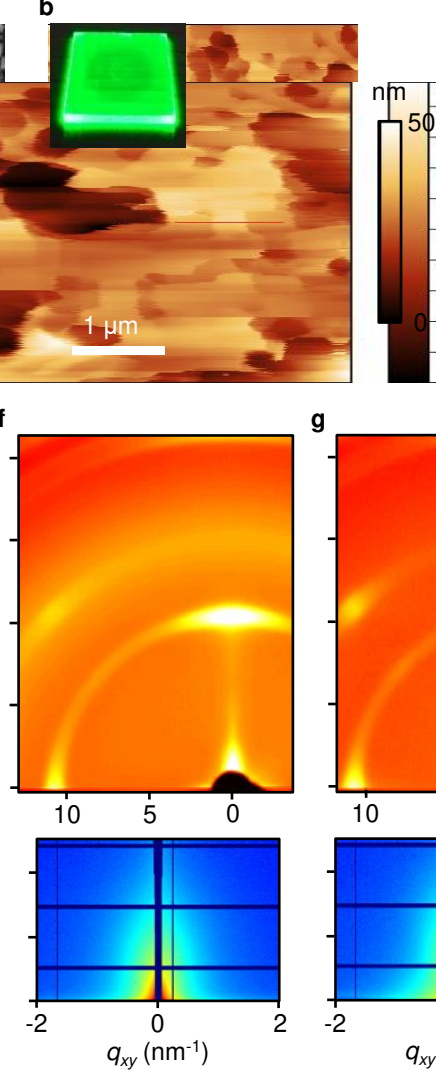
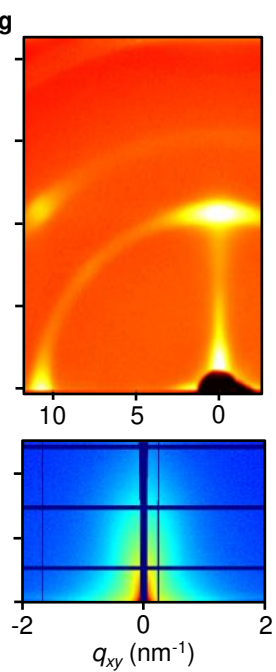

C
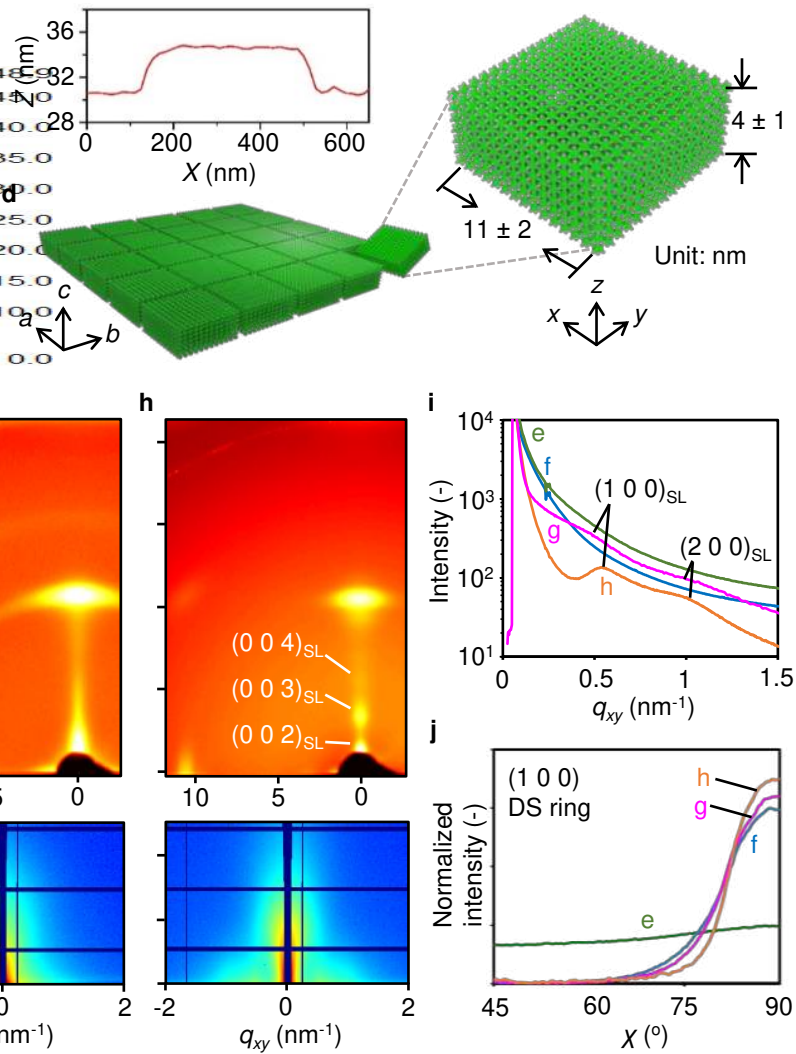

Fig. 2 | Self-assembly engineering for controlling the formation of NPL superlattices. a, TEM image and photograph of colloidal solution exposed to UV light for the synthesized NPLs. b, Representative AFM height image and photograph exposed to UV light for the fabricated NPL superlattice film in g. c. Cross-sectional height profile corresponding to the black line in b, revealing laterally $2 \mathrm{D}$ assembly of NPLs in the $x y$ (substrate) plane. $\mathbf{d}$, Schematics of NPL 2DSL showing the superlattice $a b$ plane coincides with the $x y$ plane and the SL $c$ axis in parallel to the $z$ vector. e-h, GIWAXS (top) and GISAXS (bottom) patterns for the drop-casted film (e), spin-coated film on poly-TPD (f), spin-coated film on low-surface-energy X-F4 TAPC $(\mathbf{g})$, and spin-coated film on X-F4 TAPC comprising NPLs synthesized using OLA ligands (h). The Miller index $(a b c)_{s L}$ refers to a superlattice plane. i, $q_{x y}$ cuts for the GISAXS patterns in panels e to h. j, Comparison of orientation distribution function for the emissive films considered here using the normalized scattering intensity of the (100) DS ring with respect to $\chi$, revealing an enhanced horizontal orientation upon 2DSL formation.

We hypothesized that the NPL stack ordering is highly influenced by the inter-NC interactions. In order to minimize the effect of underlying substrate, we examined the spin-coated NPL films sitting on a fluorinated hole transport material (HTM), X-F6-TAPC. Remarkably, the lowsurface-energy surface was found to further enhance NPL ordering by forming twodimensional superlattices (2DSLs). The atomic force microscope (AFM) height image (Figs. $2 \mathrm{~b}$ and $2 \mathrm{c}$ and Supplementary Fig. 4) reveals the formation of square 2DSLs comprised of close-packed NPL arrays with the side faces linked to each other, having the thickness of 3 $4 \mathrm{~nm}$ and lateral dimension exceeding $300 \mathrm{~nm}$. The 2DSL $c$ axis coincides with the NPL OP vector. We estimate that the emissive layer $(E M L)$ in device contains in average $8-10$ stacking 
layers of 2DSL. The GIWAXS pattern highlights the extension of a SL Bragg rod along the $q_{z}$ axis, and the SL peaks corresponding to lateral packing, denoted as (100)SL and (200)SL, come out on the $q_{x y}$ axis in the GISAXS pattern (Figs. $2 g$ and $2 \mathrm{i}$ ). Hereafter, we optimized our LED device based on the 2DSL-contained EMLs.

Note that we observed an even higher SL crystallinity by replacing DA with oleylamine (OLA) in our synthetic protocol (Fig. 2h), as reflected by the emergence of SL Bragg peaks on the $q_{z}$ axis, corresponding to $(00 / \mathrm{SL}$, where $/$ is an integer. Figure 2j compares the (100) DS ring intensity as a function of polar angle $\chi$ for the four samples considered here, revealing the improvement of horizontal NPL orientation upon 2DSL formation. Nevertheless, we were not able to reach high electroluminescent (EL) efficiencies with the OLA-based NPLs, hypothetically due to the long hydrophobic tail. More discussions about photophysical properties for the emissive thin films see Supplementary Section 2.1.

Analysis of Thin-Film Radiation Patterns. The thin-film stack (air/EML/X-F6-TAPC/glass) was mounted on a hemicylindrical glass prism using a refractive index matching optical liquid, followed by performing the polarization- and angle-dependent PL spectroscopy ${ }^{47}$ that differentiates between the $s$-polarized ( $s$-pol) emission from the transverse-electric $y$ dipoles and the $p$-polarized ( $p$-pol) emission from the transverse-magnetic $x$ and $z$ dipoles. The film thickness, $t$, and refractive index, $n$, for each dielectric layer was carefully characterized by ellipsometry (Supplementary Section 2.2). The generated radiation pattern resolves the PL intensity, $I$, on the substrate plane $(x-y)$ projection of emission wave vector $k, k_{x}$ and $k_{y}$, which inform the effective TDM orientation in the EML within the $k$-space domain, $k / k_{0}<n_{\text {glass }}$, where $k_{0}$ is the wave vector in air and $n_{\text {glass }}=1.52$ is the refractive index of glass. It follows that $k / k_{0}$ $=1$ corresponds to the critical angle of total internal reflection (TIR) at the glass/air interface. Since the $p$-pol emission comes from both horizontal and vertical dipoles, optical simulations were carried out to fit the $p$-pol profile to quantify the dipole orientation, using the thin-film horizontal dipole ratio, $\Theta_{\mathrm{H}}$, as the only fitting parameter. ${ }^{25}$

Figure 3a presents the experimentally measured $p$-pol PL intensity as a function of viewing angle, $\phi$, together with the calculated profiles for $\Theta_{\mathrm{H}}$ values of $0.67,0.72$, and 0.82 , corresponding to isotropic, best-fitted, and AR $\rightarrow \infty$ models, respectively. Indeed, as revealed in Fig. 1c, near the critical angle for the glass-air interface, $\phi \sim 41^{\circ}$, the emission from a horizontal dipole nearly vanishes, while a vertical dipole strongly couples into the substrate, so the emission gets intensified. As a result, a lower $\Theta_{\mathrm{H}}$ would lead to a shallower minimum at $\phi \sim 41^{\circ}$ and a higher fraction of light coupled into the substrate mode, $\phi>41^{\circ}$. Figure $3 \mathrm{~b}$ compares the experimentally measured and theory-fitted $k$-space radiation patterns, showing excellent agreement. Remarkably, the theory-fitted thin-film $\Theta_{H}=0.72$ nicely agrees with the 
predicted $\Theta_{\mathrm{IP}}=0.74$ for individual NPL (see last section). We attribute the enhanced ratio of horizontal dipole to the formation of 2DSL that horizontally orient individual NPLs to a great degree. Consequently, the single-dipole emitter model for individual NPL (Fig. 1) is already adequate to describe the collective thin-film radiation behavior.
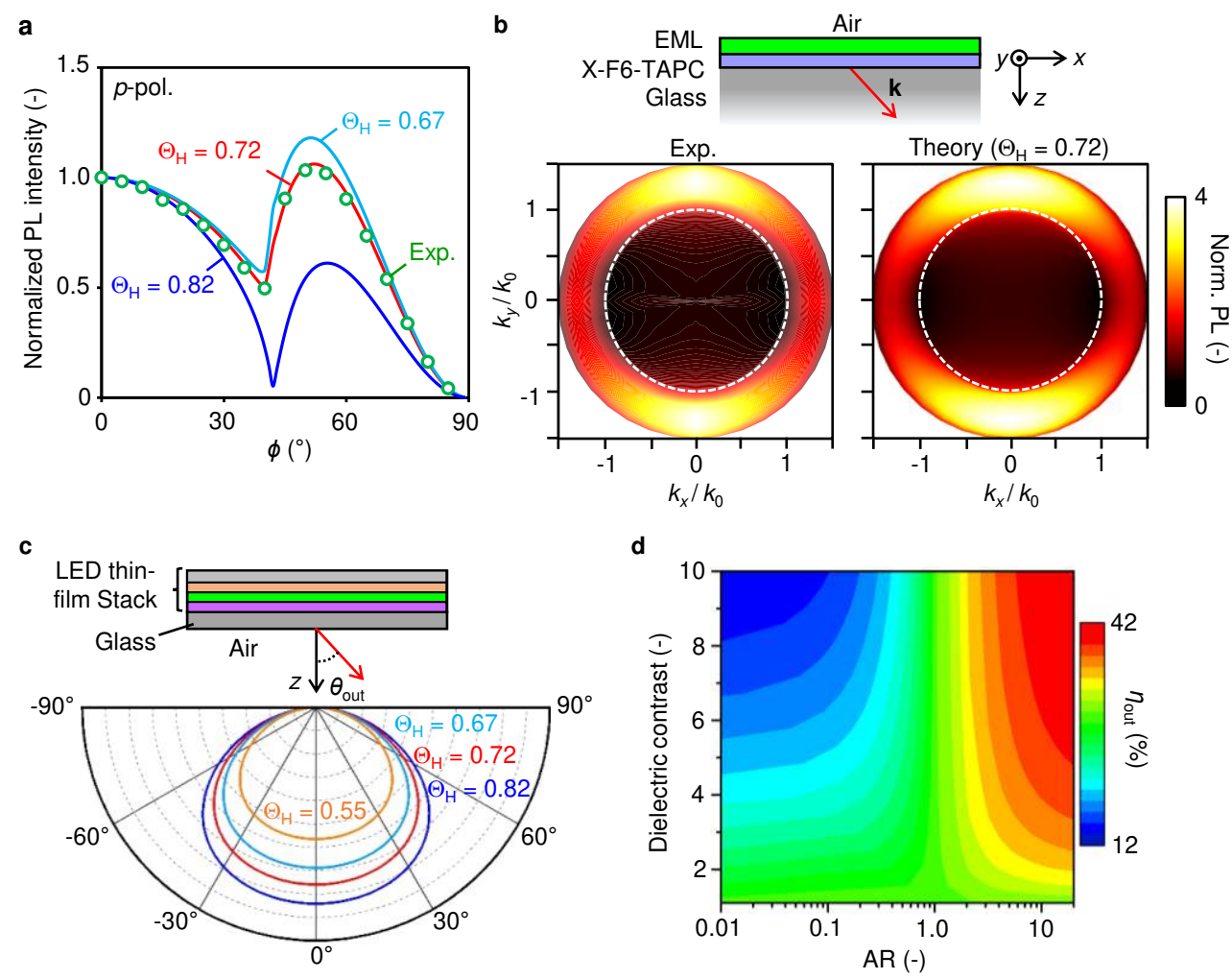

Fig. 3 | Analysis of EML radiation patterns and outcoupling efficiency calculations. a, Experimentally characterized (Exp.) and optical theory-calculated thin-film $p$-pol PL intensity as a function of viewing angle $\phi$, using the thin-film horizontal dipole ratio $\Theta_{\mathrm{H}}$ as the control parameter. b. Experimentally characterized (left) and theory-fitted (right; $\Theta_{H}=0.72$ ) thin-film $k$ space radiation patterns considering the dielectric stack of EML/X-F6-TAPC/Glass. c. Calculated far-field emission patterns (FEPs) generated by EMLs in our optimized LED stack for $\Theta_{H}$ values of $0.55,0.67,0.72$, and 0.82 , revealing a stronger radiation power outcoupled to air by increasing EML $\Theta_{\mathrm{H}}$. d, Calculated light outcoupling efficiency $\eta_{\text {out }}$ as functions of the NC aspect ratio $A R$ and dielectric contrast $\widetilde{\epsilon}$, under the assumption of thin-film $\Theta_{H}$ equal to $\Theta_{I P}$ in individual NC.

From a fundamental point of view, the combined theoretical and experimental analysis presented here highlights the importance of NC shape to the light outcoupling efficiency in QD LEDs, which has long been ignored. We further compare the calculated far-field emission patterns (FEPs) generated by EMLs in our optimized LED stack (see details in next section) for $\Theta_{H}$ values of $0.55,0.67,0.72$, and 0.82 (Fig. 3c). The $\Theta=0.55$ model corresponds to 
emission in asymptotic vertical rod NC with $\mathrm{AR} \rightarrow 0$, the lower theoretical limit for NC solids (Supplementary Section 1.2). Regardless of $\Theta_{\mathrm{H}}$ values, the calculated FEPs exhibit similarity solutions of the Lambertian function, and as expected, the radiation power increases with $\Theta_{H}$, thereby enhancing $\eta_{\text {out }}$ at device level. Accordingly, under the assumption that the thin-film $\Theta_{H}$ is equal to $\Theta_{\mathrm{IP}}$ in individual NC, Fig. 3d presents the calculated $\eta_{\text {out }}$ as functions of $A R$ and $\widetilde{\epsilon}$. Clearly, as illustrated in Fig. 1, increasing dielectric contrast and aspect ratio would result in stronger emission polarization, effectively enhancing emission from the IP dipoles. Given a realistic range for the dielectric contrast in most semiconductor QDs, $\widetilde{\epsilon}<6$, we predict that highest attainable $\eta_{\text {out }}$ for QD solid-based device is approximately $40 \%$, double the efficiency from an isotropic emitter. As for the LHP NPLs considered here (AR 2.75 and $\widetilde{\epsilon} \sim 2.12$ ), the theoretical $\eta_{\text {out }}$ is $\sim 30 \%$.

LED Fabrication and Characterization. The spin-coated NPL thin films were directly employed as EMLs to examine the EL performance. We first optimized the electron transport layer (EML) material and process based on the device architecture of ITO/PEDOT:PSS/EML/ETL/LiF/AI (for full compound names see Methods). The control set of devices that used standard TPBi as ETL exhibited relatively modest peak external quantum efficiency, $\eta_{\text {ext }}$, of $5.87 \%$ and current efficiency, $\eta_{\mathrm{CE}}$, of $24.74 \mathrm{~cd} \mathrm{~A}^{-1}$. By replacing TPBi with 3TPYMB, the peak $\eta_{\text {ext }}\left(\eta_{\mathrm{CE}}\right)$ was enhanced to $10.6 \%\left(46.6 \mathrm{~cd} \mathrm{~A}^{-1}\right)$, with the turn-on voltage, $V_{\text {on }}$, down to $2.75 \mathrm{~V}$. Other ETL materials were also tested but did not give better performance (see Supplementary Table 3). We attribute the observed efficiency enhancement to its low electron injection barrier and relatively low refractive index, $n=1.65$, which increases $\eta_{\text {out }}$ by reducing the substrate-mode and surface-plasmon-polariton losses. ${ }^{48-50}$

We next optimized the hole transport layer $(\mathrm{HTL})$ material and process based on the device architecture of ITO/PEDOT:PSS/HTL/EML/ETL/Liq/Al. Note that colloidal NPLs were directly spin-coated onto HTL, so the choice of HTL directly influences the behavior of NPL assembly. Numerous HTL materials were examined, while here we specifically compare three cases, without HTL, poly-TPD, and X-F6-TAPC, in which the last induces the formation of 2DSLs (see Fig. 2). As for the X-F6-TAPC devices, after extensive device optimization, the following device architecture was developed: ITO (120 $\pm 15 \mathrm{~nm}$ ) / PEDOT:PSS (35 nm) / X-F6-TAPC (18 nm) / EML (32 nm) / 3TPYMB (50 nm) / Liq (3 nm) / Al (70 nm). The device cross-sectional TEM image and energy diagram are shown in Figs. 4a-b. Figures 4c-d present the current density, $J$, and luminance, $L$, as a function of voltage, $V$, for the three systems considered here. Angledependent EL measurement revealed that the radiation patterns are excellently Lambertian (Supplementary Fig. 12). The full angular EL distribution allow us to calculate $\eta_{\text {ext }}$ as a function of $L$ (Fig. 4e). As compared to the HTL-free devices, the insertion of thin (19 $\pm 2 \mathrm{~nm}$ ) poly-TPD 
layer between ETL and PEDOT:PSS greatly enhanced the driving current and luminance, but the efficiencies slightly dropped.

In the X-F6-TAPC devices, although the low-hole-mobility nature slightly impedes hole transport, ${ }^{51}$ the peak $\eta_{\text {ext }}$ is impressively improved up to $24.2 \%$ (Fig. 3e), which to our knowledge, represents the highest external quantum efficiency ever reported in solutionprocessed QD LEDs based on all semiconductor materials. ${ }^{4,-9,52-58}$ In another device, the peak $\eta_{\mathrm{CE}}$ and power efficiency, $\eta_{\mathrm{PE}}$, reach $107.5 \mathrm{~cd} \mathrm{~A}^{-1}$ and $112.5 \mathrm{Im} \mathrm{W}^{-1}$ (Supplementary Fig. 13), respectively, comparable to the state-of-the-art OLEDs. We notice that the high efficiency values were obtained without using any defects passivating techniques. The statistical analysis (out of 54 devices) of peak $\eta_{\text {ext }}$ is shown in Fig. $4 \mathrm{f}$. The EL emission maximum and full width at half maximum (fwhm) are $528 \pm 1$ and $22 \pm 2 \mathrm{~nm}$, respectively, yielding high-color-purity green chromaticity fulfilling Rec. 2020 color gamut. Moreover, the high-efficiency range is wide, with $\eta_{\text {ext }}>20 \%$ between 30 to $1500 \mathrm{~cd} \mathrm{~m}^{-2}$, nicely covering the brightness range of interest in commercial displays. A degree of efficiency roll-off at high luminance $\left(\eta_{\text {ext }}\left(5009 \mathrm{~cd} \mathrm{~m}^{-2}\right)=\right.$ $10.9 \%)$, nevertheless, was still observed.

a

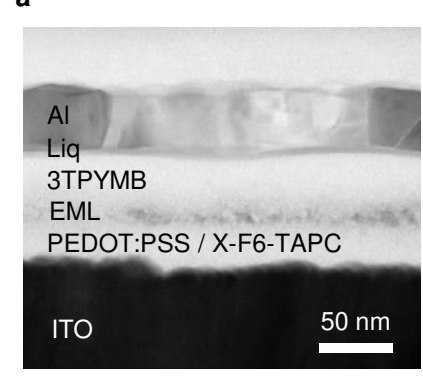

e

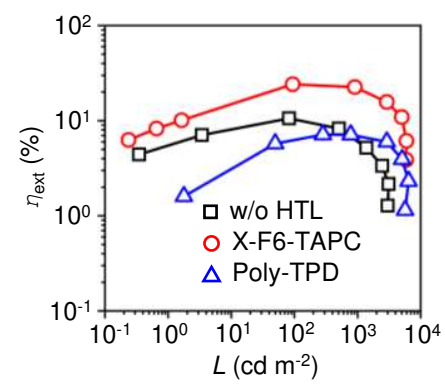

b
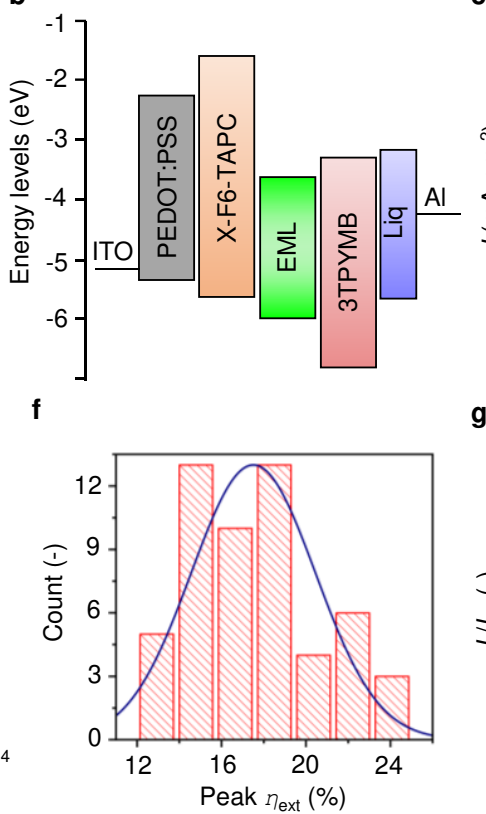
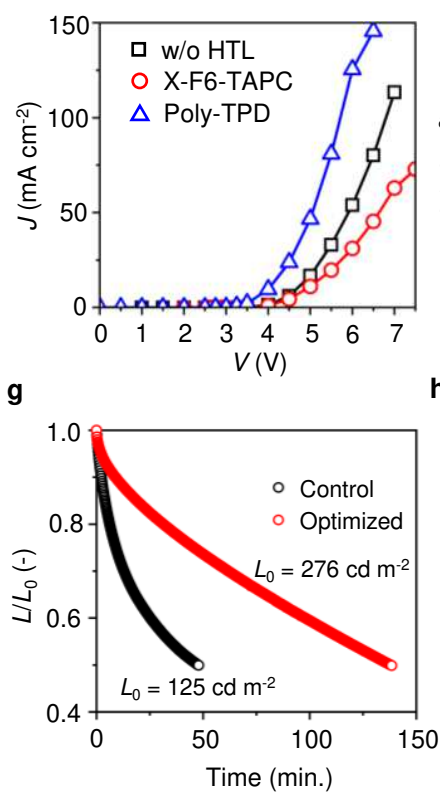

d

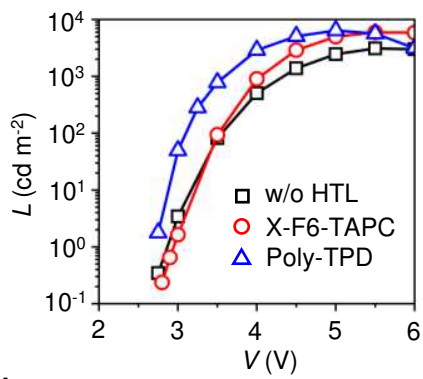

h

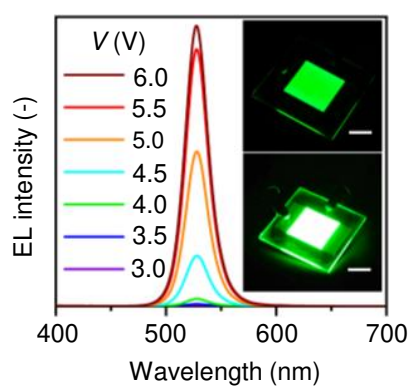

Fig. 4 | Device characteristics of 2DSL-based LEDs. a-b, Representative TEM image (a) and energy diagram (b) of our optimized device architecture, using the EML comprised of 2DSLs of NPLs. c-e, Optimized LED current density $J(\mathbf{c})$, luminance $L(\mathbf{d})$, and external quantum efficiency $\eta_{\text {ext }}$ as a function of $V$ for the devices of (i) without (w/o) HTL, (ii) X-F6TAPC HTL, and (iii) poly-TPD HTL. f, Statistical distribution of peak LED $\eta_{\text {ext }}$ out of 54 devices. g, Relative luminance as a function of time under continuous electrical stress at a constant current density of $0.5 \mathrm{~mA} \mathrm{~cm}{ }^{-2}$, corresponding to initial luminance $L_{0}$ of 276 and $125 \mathrm{~cd} \mathrm{~m}^{-2}$ for the optimized and control devices, respectively. $\mathbf{h}$, EL spectra and photographs for our largearea LEDs. 
In addition to the formation of 2DSL that preserves the preferentially in-plane dipole orientation in individual square NPLs, as discussed in Figs. 1-3, the following factors also contribute to the enhanced external quantum efficiency, including (i) near-unity thin-film $\eta_{\mathrm{PL}}$, (ii) cascade highest-occupied-molecular-orbital (HOMO) energy levels that facilitate hole injection, ${ }^{50,51}$ (iii) high lowest-unoccupied-molecular-orbital (LUMO) level of X-F6-TAPC that effectively confines injected electrons within the EML, ${ }^{50,51,59}$ and (iv) low refractive indices for ETL, HTL, and EML that boost $\eta_{\text {out }}$ (details see Supplementary Section 2).

We also investigated operational lifetime for our X-F6-TAPC-based devices, as compared with the control device (w/o HTL). The time for the luminance to decay to $50 \%$ of the initial luminance, $\mathrm{LT}_{50}$, under a constant current density of $0.5 \mathrm{~mA} \mathrm{~cm}{ }^{-2}$, corresponding to the initial luminance, $L_{0}$, of 276 and $125 \mathrm{~cd} \mathrm{~m}^{-2}$ for the optimized and control devices, respectively. The optimized device exhibits $\mathrm{LT}_{50}$ of 138 minutes, threefold higher than that for control device (details see Supplementary Section 3). The EL spectra remained consistent throughout the stability tests (Supplementary Fig. 15). A large-area $\left(225 \mathrm{~mm}^{2}\right)$ device was also demonstrated without significant compromise of device performance (Fig. 4h and Supplementary Fig. 16).

\section{Conclusions}

We have theoretically and experimentally demonstrated that the two-dimensional superlattices of high-aspect-ratio LHP nanoplatelets preserve the effective in-plane dipole orientation in individual nanocrystal, thereby boosting the intrinsic light outcoupling efficiency in LEDs. Our findings lay the foundation for future QD shape and assembly engineering. In combination with the emerging defect passivating techniques, we anticipate that the performance gap between QD LEDs and OLEDs will be closed in the near future. 


\section{Acknowledgement}

C.J.S., S.K, and T.M. are grateful for financial support from the ETH research grant (ETH-33 18-2), Swiss National Science Foundation project grant (project number: 200021-178944), and European Research Council (ERC) starting grant (N849229 - CQWLED). In addition, the technical support from the FIRST Lab in ETH Zurich is highly appreciated. The authors also appreciate technical support of Reuteler Joakim and Zeng Peng from ScopeM, the Scientific Center for Optical and Electron Microscopy at ETH Zürich. The authors declare that they have no other competing interests. Data and materials availability: All data needed to evaluate the conclusions in the paper are present in the paper and/or Supporting Information. Additional data related to this paper may be requested from the authors.

\section{Author contribution}

S.K., T.M., and C.J.S. conceived the idea and designed the experiments. S.K. synthesized CQWs and carried out their photophysical, optical, and morphological characterization. S.K. designed, fabricated, and characterized the perovskite LED devices. S.K. and T.M. performed optical simulations. T.M. performed most theoretical analysis. Y.T.L. performed GISAXS under supervision of Y.C.C. F.K acquired cryo TEM images of perovskite NCs. S.K, T.M., and C.J.S. co-wrote the paper. All authors contributed to this work, commented on the paper, and agreed to the contents of the paper and supplementary materials.

\section{Corresponding authors}

All correspondences regarding this paper should be addressed to Chih-Jen Shih at chihjen.shih@chem.ethz.ch.

\section{Competing interests}

The authors declare no competing interests.

\section{Supplementary information}

Supplementary Sections 1-3, Figs. 1-18, and Tables 1-3. 


\section{References}

1 Coe, S., Woo, W.-K., Bawendi, M. \& Bulović, V. Electroluminescence from single monolayers of nanocrystals in molecular organic devices. Nature 420, 800-803, doi:10.1038/nature01217 (2002).

2 Qian, L., Zheng, Y., Xue, J. \& Holloway, P. H. Stable and efficient quantum-dot light-emitting diodes based on solution-processed multilayer structures. Nature Photonics 5, 543-548, doi:10.1038/nphoton.2011.171 (2011).

3 Mashford, B. S. et al. High-efficiency quantum-dot light-emitting devices with enhanced charge injection. Nature Photonics 7, 407-412, doi:10.1038/nphoton.2013.70 (2013).

4 Dai, X. et al. Solution-processed, high-performance light-emitting diodes based on quantum dots. Nature 515, 96-99, doi:10.1038/nature13829 (2014).

5 Shirasaki, Y., Supran, G. J., Bawendi, M. G. \& Bulović, V. Emergence of colloidal quantum-dot light-emitting technologies. Nature Photonics 7, 13-23, doi:10.1038/nphoton.2012.328 (2013).

6 Won, Y.-H. et al. Highly efficient and stable InP/ZnSe/ZnS quantum dot light-emitting diodes. Nature 575, 634-638, doi:10.1038/s41586-019-1771-5 (2019).

7 Chiba, T. et al. Anion-exchange red perovskite quantum dots with ammonium iodine salts for highly efficient light-emitting devices. Nature Photonics 12, 681-687, doi:10.1038/s41566-0180260-y (2018).

8 Dong, Y. et al. Bipolar-shell resurfacing for blue LEDs based on strongly confined perovskite quantum dots. Nature Nanotechnology 15, 668-674, doi:10.1038/s41565-020-0714-5 (2020).

9 Kim, Y.-H. et al. Comprehensive defect suppression in perovskite nanocrystals for highefficiency light-emitting diodes. Nature Photonics, doi:10.1038/s41566-020-00732-4 (2021).

10 Sutherland, B. R. \& Sargent, E. H. Perovskite photonic sources. Nature Photonics 10, 295, doi:10.1038/nphoton.2016.62 (2016).

11 Liu, X.-K. et al. Metal halide perovskites for light-emitting diodes. Nature Materials 20, 10-21, doi:10.1038/s41563-020-0784-7 (2021).

12 Quan, L. N. et al. Perovskites for Next-Generation Optical Sources. Chemical Reviews 119, 7444-7477, doi:10.1021/acs.chemrev.9b00107 (2019).

13 Kim, Y.-H., Cho, H. \& Lee, T.-W. Metal halide perovskite light emitters. Proceedings of the National Academy of Sciences 113, 11694-11702, doi:10.1073/pnas.1607471113 (2016).

$14 \mathrm{Li}, \mathrm{G}$. et al. Surface Ligand Engineering for Near-Unity Quantum Yield Inorganic Halide Perovskite QDs and High-Performance QLEDs. Chemistry of Materials 30, 6099-6107, doi:10.1021/acs.chemmater.8b02544 (2018).

15 Pan, J. et al. Bidentate Ligand-Passivated CsPbl3 Perovskite Nanocrystals for Stable Near-Unity Photoluminescence Quantum Yield and Efficient Red Light-Emitting Diodes. Journal of the American Chemical Society 140, 562-565, doi:10.1021/jacs.7b10647 (2018).

16 Di Stasio, F., Christodoulou, S., Huo, N. \& Konstantatos, G. Near-Unity Photoluminescence Quantum Yield in CsPbBr3 Nanocrystal Solid-State Films via Postsynthesis Treatment with Lead Bromide. Chemistry of Materials 29, 7663-7667, doi:10.1021/acs.chemmater.7b02834 (2017). Dutta, A., Behera, R. K., Pal, P., Baitalik, S. \& Pradhan, N. Near-Unity Photoluminescence Quantum Efficiency for All CsPbX3 ( $\mathrm{X}=\mathrm{Cl}, \mathrm{Br}$, and I) Perovskite Nanocrystals: A Generic Synthesis Approach. Angewandte Chemie International Edition 58, 5552-5556, doi:https://doi.org/10.1002/anie.201900374 (2019).

18 Zhong, Q. et al. L-Type Ligand-Assisted Acid-Free Synthesis of CsPbBr3 Nanocrystals with NearUnity Photoluminescence Quantum Yield and High Stability. Nano Letters 19, 4151-4157, doi:10.1021/acs.nanolett.9b01666 (2019).

19 Yablonovitch, E. Statistical ray optics. J. Opt. Soc. Am. 72, 899-907, doi:10.1364/JOSA.72.000899 (1982).

20 Cho, C. et al. The role of photon recycling in perovskite light-emitting diodes. Nature Communications 11, 611, doi:10.1038/s41467-020-14401-1 (2020). 
21 Eaton, S. W. et al. Lasing in robust cesium lead halide perovskite nanowires. Proceedings of the National Academy of Sciences 113, 1993, doi:10.1073/pnas.1600789113 (2016).

22 Ndione, P. F., Li, Z. \& Zhu, K. Effects of alloying on the optical properties of organic-inorganic lead halide perovskite thin films. Journal of Materials Chemistry C 4, 7775-7782, doi:10.1039/C6TC02135B (2016).

23 Kim, K.-H. et al. Phosphorescent dye-based supramolecules for high-efficiency organic lightemitting diodes. Nature Communications 5, 4769, doi:10.1038/ncomms5769 (2014).

24 Kim, S.-Y. et al. Organic Light-Emitting Diodes with 30\% External Quantum Efficiency Based on a Horizontally Oriented Emitter. Advanced Functional Materials 23, 3896-3900, doi:https://doi.org/10.1002/adfm.201300104 (2013).

25 Kim, K.-H. \& Kim, J.-J. Origin and Control of Orientation of Phosphorescent and TADF Dyes for High-Efficiency OLEDs. Advanced Materials 30, 1705600, doi:https://doi.org/10.1002/adma.201705600 (2018).

26 Gather, M. \& Reineke, S. Recent advances in light outcoupling from white organic lightemitting diodes. Journal of Photonics for Energy 5, 057607 (2015).

27 Koo, W. H. et al. Light extraction from organic light-emitting diodes enhanced by spontaneously formed buckles. Nature Photonics 4, 222-226, doi:10.1038/nphoton.2010.7 (2010).

28 Sun, Y. \& Forrest, S. R. Enhanced light out-coupling of organic light-emitting devices using embedded low-index grids. Nature Photonics 2, 483-487, doi:10.1038/nphoton.2008.132 (2008).

29 Do, Y. R., Kim, Y.-C., Song, Y.-W. \& Lee, Y.-H. Enhanced light extraction efficiency from organic light emitting diodes by insertion of a two-dimensional photonic crystal structure. Journal of Applied Physics 96, 7629-7636, doi:10.1063/1.1815049 (2004).

30 Gifford, D. K. \& Hall, D. G. Emission through one of two metal electrodes of an organic lightemitting diode via surface-plasmon cross coupling. Applied Physics Letters 81, 4315-4317, doi:10.1063/1.1525882 (2002).

31 Schuller, J. A. et al. Orientation of luminescent excitons in layered nanomaterials. Nature Nanotechnology 8, 271-276, doi:10.1038/nnano.2013.20 (2013).

32 Echeverry, J. P., Urbaszek, B., Amand, T., Marie, X. \& Gerber, I. C. Splitting between bright and dark excitons in transition metal dichalcogenide monolayers. Physical Review B 93, 121107, doi:10.1103/PhysRevB.93.121107 (2016).

33 Amani, M. et al. Near-unity photoluminescence quantum yield in MoS\&lt;sub\&gt;2\&lt;/sub\&gt. Science 350, 1065, doi:10.1126/science.aad2114 (2015).

34 Scott, R. et al. Directed emission of CdSe nanoplatelets originating from strongly anisotropic 2D electronic structure. Nature Nanotechnology 12, 1155-1160, doi:10.1038/nnano.2017.177 (2017).

35 Ma, X. et al. Anisotropic Photoluminescence from Isotropic Optical Transition Dipoles in Semiconductor Nanoplatelets. Nano Letters 18, 4647-4652, doi:10.1021/acs.nanolett.8b00347 (2018).

36 Gao, Y., Weidman, M. C. \& Tisdale, W. A. CdSe Nanoplatelet Films with Controlled Orientation of their Transition Dipole Moment. Nano Letters 17, 3837-3843, doi:10.1021/acs.nanolett.7b01237 (2017).

37 Tanaka, K. et al. Comparative study on the excitons in lead-halide-based perovskite-type crystals $\mathrm{CH} 3 \mathrm{NH} 3 \mathrm{PbBr} 3 \mathrm{CH} 3 \mathrm{NH} 3 \mathrm{Pbl}$. Solid State Communications 127, 619-623, doi:https://doi.org/10.1016/S0038-1098(03)00566-0 (2003).

38 Becker, M. A. et al. Bright triplet excitons in caesium lead halide perovskites. Nature 553, 189193, doi:10.1038/nature25147 (2018).

$39 \mathrm{Hu}$, J. et al. Linearly Polarized Emission from Colloidal Semiconductor Quantum Rods. Science 292, 2060, doi:10.1126/science.1060810 (2001). 

Perovskite Nanocrystals. Nano Letters 17, 4534-4540, doi:10.1021/acs.nanolett.7b02147 (2017).

41 Jurow, M. J. et al. Manipulating the Transition Dipole Moment of CsPbBr3 Perovskite Nanocrystals for Superior Optical Properties. Nano Letters 19, 2489-2496, doi:10.1021/acs.nanolett.9b00122 (2019).

42 Jagielski, J. et al. Scalable photonic sources using two-dimensional lead halide perovskite superlattices. Nature Communications 11, 387, doi:10.1038/s41467-019-14084-3 (2020).

43 Lee, T. et al. Controlling the dimension of the quantum resonance in CdTe quantum dot superlattices fabricated via layer-by-layer assembly. Nature Communications 11, 5471, doi:10.1038/s41467-020-19337-0 (2020).

44 Wilson, J. N., Frost, J. M., Wallace, S. K. \& Walsh, A. Dielectric and ferroic properties of metal halide perovskites. APL Materials 7, 010901, doi:10.1063/1.5079633 (2019).

45 Kumar, S. et al. Efficient Blue Electroluminescence Using Quantum-Confined Two-Dimensional Perovskites. ACS Nano 10, 9720-9729, doi:10.1021/acsnano.6b05775 (2016).

46 Kumar, S., Jagielski, J., Marcato, T., Solari, S. F. \& Shih, C.-J. Understanding the Ligand Effects on Photophysical, Optical, and Electroluminescent Characteristics of Hybrid Lead Halide Perovskite Nanocrystal Solids. The Journal of Physical Chemistry Letters 10, 7560-7567, doi:10.1021/acs.jpclett.9b02950 (2019).

47 Frischeisen, J., Yokoyama, D., Adachi, C. \& Brütting, W. Determination of molecular dipole orientation in doped fluorescent organic thin films by photoluminescence measurements. Applied Physics Letters 96, 073302, doi:10.1063/1.3309705 (2010).

48 Salehi, A. et al. Highly Efficient Organic Light-Emitting Diode Using A Low Refractive Index Electron Transport Layer. Advanced Optical Materials 5, 1700197, doi:https://doi.org/10.1002/adom.201700197 (2017).

49 Shin, H. et al. Sky-Blue Phosphorescent OLEDs with 34.1\% External Quantum Efficiency Using a Low Refractive Index Electron Transporting Layer. Advanced Materials 28, 4920-4925, doi:https://doi.org/10.1002/adma.201506065 (2016).

50 Jou, J.-H., Kumar, S., Agrawal, A., Li, T.-H. \& Sahoo, S. Approaches for fabricating high efficiency organic light emitting diodes. Journal of Materials Chemistry C 3, 2974-3002, doi:10.1039/C4TC02495H (2015).

51 Liaptsis, G. \& Meerholz, K. Crosslinkable TAPC-Based Hole-Transport Materials for SolutionProcessed Organic Light-Emitting Diodes with Reduced Efficiency Roll-Off. Advanced Functional Materials 23, 359-365, doi:https://doi.org/10.1002/adfm.201201197 (2013).

52 Yeom, J. E. et al. Good Charge Balanced Inverted Red InP/ZnSe/ZnS-Quantum Dot LightEmitting Diode with New High Mobility and Deep HOMO Level Hole Transport Layer. ACS Energy Letters 5, 3868-3875, doi:10.1021/acsenergylett.0c02193 (2020).

53 Yan, F. et al. Highly Efficient Visible Colloidal Lead-Halide Perovskite Nanocrystal Light-Emitting Diodes. Nano Letters 18, 3157-3164, doi:10.1021/acs.nanolett.8b00789 (2018).

$54 \mathrm{Kim}, \mathrm{T}$. et al. Efficient and stable blue quantum dot light-emitting diode. Nature 586, 385-389, doi:10.1038/s41586-020-2791-x (2020).

55 Wang, L. et al. Blue Quantum Dot Light-Emitting Diodes with High Electroluminescent Efficiency. ACS Applied Materials \& Interfaces 9, 38755-38760, doi:10.1021/acsami.7b10785 (2017).

56 Cao, Y. et al. Perovskite light-emitting diodes based on spontaneously formed submicrometrescale structures. Nature 562, 249-253, doi:10.1038/s41586-018-0576-2 (2018).

57 Lin, K. et al. Perovskite light-emitting diodes with external quantum efficiency exceeding 20 per cent. Nature 562, 245-248, doi:10.1038/s41586-018-0575-3 (2018).

58 Hassan, Y. et al. Ligand-engineered bandgap stability in mixed-halide perovskite LEDs. Nature 591, 72-77, doi:10.1038/s41586-021-03217-8 (2021). 
59 Daisaku, T., Takashi, T., Takayuki, C., Soichi, W. \& Junji, K. Novel Electron-transport Material Containing Boron Atom with a High Triplet Excited Energy Level. Chemistry Letters 36, 262263, doi:10.1246/cl.2007.262 (2007). 


\section{Supplementary Files}

This is a list of supplementary files associated with this preprint. Click to download.

- Methods.pdf

- Supplementarylnformationv320210513.pdf 